\title{
Expression of nephrin in the human placenta and fetal membranes
}

\author{
BO HYON YUN ${ }^{1}$, SEUNG MI LEE ${ }^{2}$, HEE YOUNG CHO ${ }^{1}$, JI YOUNG KIM ${ }^{3}$, GA HYUN SON $^{4}$, \\ YOUNG HAN KIM ${ }^{1}$, YONG WON PARK ${ }^{1}$, BEOM JIN LIM $^{5}$ and JA YOUNG KWON ${ }^{1}$ \\ ${ }^{1}$ Division of Maternal-Fetal Medicine, Department of Obstetrics and Gynecology, Institute of Women's Life Medical Science, \\ Yonsei University College of Medicine, Seoul 120-752; ${ }^{2}$ Department of Obstetrics and Gynecology, \\ Seoul National University College of Medicine, Seoul 110-799; ${ }^{3}$ Department of Dermatology, Yonsei University College of Medicine, \\ Seoul 120-752; ${ }^{4}$ Department of Obstetrics and Gynecology, Kangnam Sacred Heart Hospital, Hallym University Medical Center, \\ Seoul 150-719; ${ }^{5}$ Department of Pathology, Gangnam Severance Hospital, Yonsei University Health System, \\ Seoul 135-720, Republic of Korea
}

Received March 10, 2014; Accepted November 20, 2014

DOI: $10.3892 / \mathrm{mmr} .2015 .4044$

\begin{abstract}
Nephrin is the signature molecule in the podocyte of the glomerulus that forms the renal slit diaphragm, the main functional unit of the glomerulus. The present study focused on the expression of nephrin in the human placenta, which may also have a role in filtration and the maintenance of homeostasis in the kidneys. A total of nine placentas from normal healthy pregnant females at full term were investigated. Reverse transcription-quantitative polymerase chain reaction, western blotting and immunofluorescence were performed. The expression of nephrin mRNA was relatively increased in the chorion compared with that in the villi and the amnion. The nephrin gene was detected in the villous cytotrophoblast cells and the endothelium of the intravillous vessels. It was also present in the chorionic and amniotic membranous lining, with its distribution being particularly dense in the amniocytes. The identification of nephrin in the human placenta, particularly at the maternal-fetal interface, provides a novel insight into the molecular basis of the selective permeability of the placental barrier, which requires further elucidation.
\end{abstract}

\section{Introduction}

The podocyte is the key component of the glomerular slit diaphragm. The podocyte and its associated proteins form the

Correspondence to: Dr Ja Young Kwon, Division of Maternal-Fetal Medicine, Department of Obstetrics and Gynecology, Institute of Women's Life Medical Science, Yonsei University College of Medicine, 50 Yonsei-ro, Seodaemun-gu, Seoul 120-752, Republic of Korea E-mail: jaykwon@yuhs.ac

Dr Beom Jin Lim, Department of Pathology, Gangnam Severance Hospital, Yonsei University Health System, 712 Eonjuro, Gangnam-gu, Seoul 135-720, Republic of Korea

E-mail: bjlim@yuhs.ac

Key words: pregnancy, placenta, nephrin, podocyte diaphragm and these cells have an intimate interaction with capillary endothelial cells, which exhibit an important role in selective filtration acting as a barrier. Previously, several studies have identified podocyte-associated proteins as the main factors in nephropathy demonstrating glomerular basement membrane alteration, such as in diabetic nephropathy $(1,2)$. Notably, the blood-brain barrier of the central nervous system, another representative homeostatic barrier system in the human body, contains astrocytes, which are similar to podocytes in structure and function $(3,4)$.

Nephrin is a podocyte-associated protein, a constituent of the slit diaphragm of the glomerulus, which appears to have a critical role in maintaining glomerular filtration. Previously, inactivation of the nephrin gene in the mouse embryo demonstrated a detrimental loss of the ultrafiltration function of the kidneys (5).

Similar to the kidney, the placenta is a major organ of the fetus, which filtrates and exchanges fetal and maternal blood, enabling the placental cotyledon and the glomerulus to undertake homeostatic functions. Notably, the expression of the nephrin gene has been reported in the fetal membranes and placenta of pregnant Sprague-Dawley rats (6). This led us to hypothesize that nephrin may be involved in regulating homeostasis at the maternal-fetal interface. However, thus far, evidence of nephrin expression in the human placenta is lacking. Therefore, the objective of the present study was to evaluate the presence and localization of nephrin, a signature molecule of podocytes (7), in the human placenta.

\section{Materials and methods}

Participants. Females that were pregnant with a single fetus, who delivered a normal infant between 37 and 40 weeks of gestation by Cesarean section at Severance Hospital, Yonsei University College of Medicine (Seoul, Republic of Korea), between January 2011 and July 2012 were enrolled in the present study. Patients with a history of active labor prior to the surgery, multiple pregnancies, prior or current diagnosis of any medical illness (diabetes, gestational diabetes, hypertension, thyroid disease or infectious disease), placenta previa, fetal anomaly, oligohydramnios, hydramnios, fetal aneuploidy, preterm labor 
Table I. Sequence of primers for GAPDH and the human nephrin gene.

\begin{tabular}{lc}
\hline Gene & Primer sequence $\left(5^{\prime}-3^{\prime}\right)$ \\
\hline GAPDH & \\
Sense & AGGCCAACCGCGAGAAGATGACC \\
Antisense & GAAGTCCAGGGCGACGTAGCAC \\
Human nephrin & \\
Sense & CCAACATCGTTTTCACTTGG \\
Antisense & GGGTGGTACGACATCCACAT \\
\hline
\end{tabular}

or premature rupture of the membranes during the present pregnancy were excluded from the present study. The present study was approved by the Institutional Review Board of Yonsei University College of Medicine and all patients consented to be involved in the present study.

Sample collection. When the placenta was delivered, the amnion and chorion were dissected under aseptic conditions. Dissected membranes were sampled at a size of $1.0 \times 1.0 \times 1.0 \mathrm{~cm}^{3}$. Villous tissue measuring $1.0 \times 1.0 \times 1.0 \mathrm{~cm}^{3}$ was sampled at the site near the cord insertion. Following the tissue sampling, the amnion, chorion and villous tissue were frozen in liquid nitrogen and stored for further analysis.

Quantification of mature $m R N A$ levels using reverse transcription-quantitative polymerase chain reaction (RT-qPCR). RNA fractions were initially isolated from the tissue samples using an RNA extraction kit (Intron Biotechnology, Inc., Gyeonggi, Korea). RNA concentrations were measured using absorbance at 260/280 nm with a SuperScript First-Strand RT kit (Invitrogen Life Technologies, Carlsbad, CA, USA). Total RNA (1 $\mu \mathrm{g})$ was converted to cDNA using oligo-dT as the primer and reverse transcriptase (Invitrogen Life Technologies). The reaction mixture $(20 \mu \mathrm{l})$ was incubated at $65^{\circ} \mathrm{C}$ for $5 \mathrm{~min}, 50^{\circ} \mathrm{C}$ for $50 \mathrm{~min}$ and $85^{\circ} \mathrm{C}$ for $5 \mathrm{~min}$. RT-qPCR was performed with Applied Biosystems 7500 real-time PCR system (Applied Biosystems Life Technologies, Foster City, CA, USA) using the Taqman ${ }^{\circledR}$ Gene Expression Assays kit (Taqman Life Technologies, Foster City, CA, USA). A total of $20 \mu \mathrm{l}$ of the RT-qPCR reaction mixture contained $2 \mu \mathrm{l}$ RT-qPCR products, $10 \mu \mathrm{l}$ Taqman ${ }^{\circledR} 2 \mathrm{X}$ universal PCR master mix and $1 \mu 1$ 20X Taqman ${ }^{\circledR}$ Gene Expression Assay mix (RT-qPCR primers). Nuclease-free water was used to adjust the final volume to $20 \mu \mathrm{l}$. The reactions were incubated in a 96-well optical plate at $95^{\circ} \mathrm{C}$ for $10 \mathrm{~min}$, followed by 40 cycles of $95^{\circ} \mathrm{C}$ for $15 \mathrm{sec}$ and $60^{\circ} \mathrm{C}$ for $60 \mathrm{sec}$. Glyceraldehyde-3-phosphate dehydrogenase $(G A P D H)$ was used as an endogenous reference gene for normalizing the results. The relative abundance of each mRNA was calculated using the comparative cycle threshold $2^{(-\Delta \Delta C t)}$ method. Individual samples were assayed in triplicate, with three independent biological replicates. The primer sequences for the endogenous control $(G A P D H)$ and nephrin are shown in Table I.

Western blotting. Nephrin expression was examined using western blotting analysis of the tissue homogenate samples. Following isolating each protein using the cell lysis buffer
Table II. Clinical characteristics of pregnancies included in the present study.

\begin{tabular}{ll}
\hline Characteristic & \multicolumn{1}{c}{ Variable } \\
\hline Maternal age (years) & $34(30-42)$ \\
Primigravida (n) & 2 \\
Nulliparity (n) & 4 \\
Gestational days & $38 \pm 3(37 \pm 3-39 \pm 1)$ \\
Cesarean section indication (n) & \\
Prior cesarean section & 6 \\
Prior myomectomy & 1 \\
Advanced maternal age & 1 \\
Cephalopelvic disproportion & 2 \\
\hline
\end{tabular}

Data are expressed as median and range.

(Cell Signaling Technology, Inc., Danvers, MA, USA), $30 \mu \mathrm{g}$ of total protein loading was used to run $8 \%$ sodium dodecyl sulfate-polyacrylamide gel electrophoresis and then transferred to polyvinylidene difluoride membranes (Millipore, Etten-Leur, The Netherlands). The blot membranes were blocked with 5\% skimmed milk in Tris-buffered saline-Tween 20 (containing $10 \mathrm{mM}$ Tris, $\mathrm{pH}$ 8.0, $150 \mathrm{mM} \mathrm{NaCl}, 0.0025 \%$ Tween-20) at room temperature for $1 \mathrm{~h}$. Subsequently, the membrane was incubated overnight at $4{ }^{\circ} \mathrm{C}$ with specific antibodies against nephrin (polyclonal rabbit anti-human, 1:1,000; \#2265; ProSci, Portway, CA, USA) and $\beta$-actin (monoclonal mouse anti-human, 1:2,000; A5441; Sigma-Aldrich, St. Louis, MO, USA). The membrane was then washed six times with TBS-T for $5 \mathrm{~min}$. Thereafter, the membrane was incubated with anti-rabbit antibody $(\mathrm{IgG}$, 1:4,000; ab6802; Abcam, Cambridge, MA, USA) and anti-mouse antibody conjugated with horseradish peroxidase (IgG, 1:5,000; ab6808; Abcam) for $1 \mathrm{~h}$ at room temperature. The membrane was washed eight times with TBS-T for $5 \mathrm{~min}$ at room temperature. The protein was visualized on the ImageQuant LAS-4000 imager using enhanced chemiluminescence (GE Healthcare, Arlington Heights, IL, USA). Relative densities for nephrin expression were normalized using tubulin expression for each sample.

Immunofluorescence (IF). Cells were fixed with acetone for 15 min and washed three times with PBS-T [PBS/0.1\% Tween-20 
A

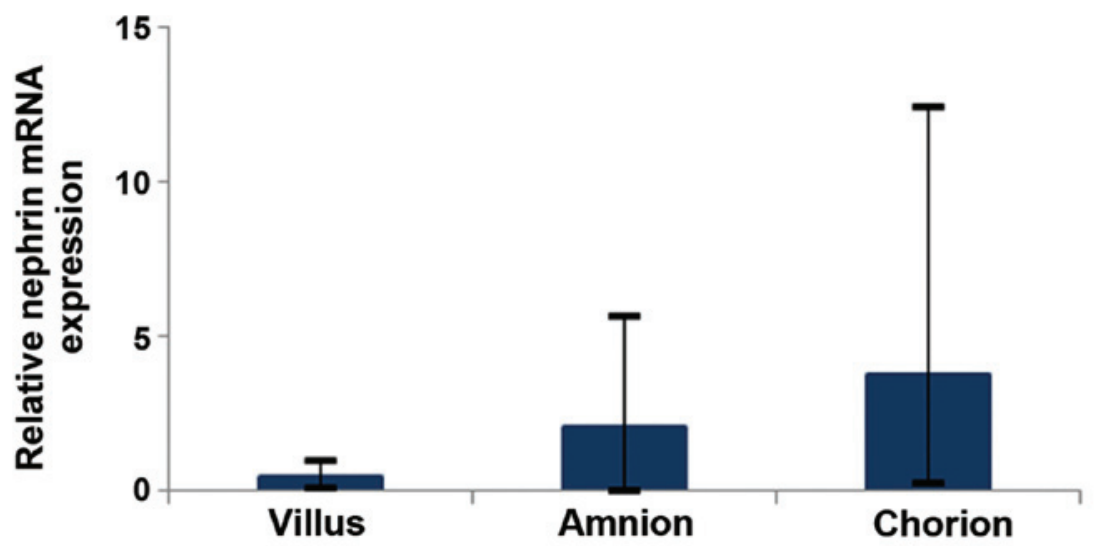

B

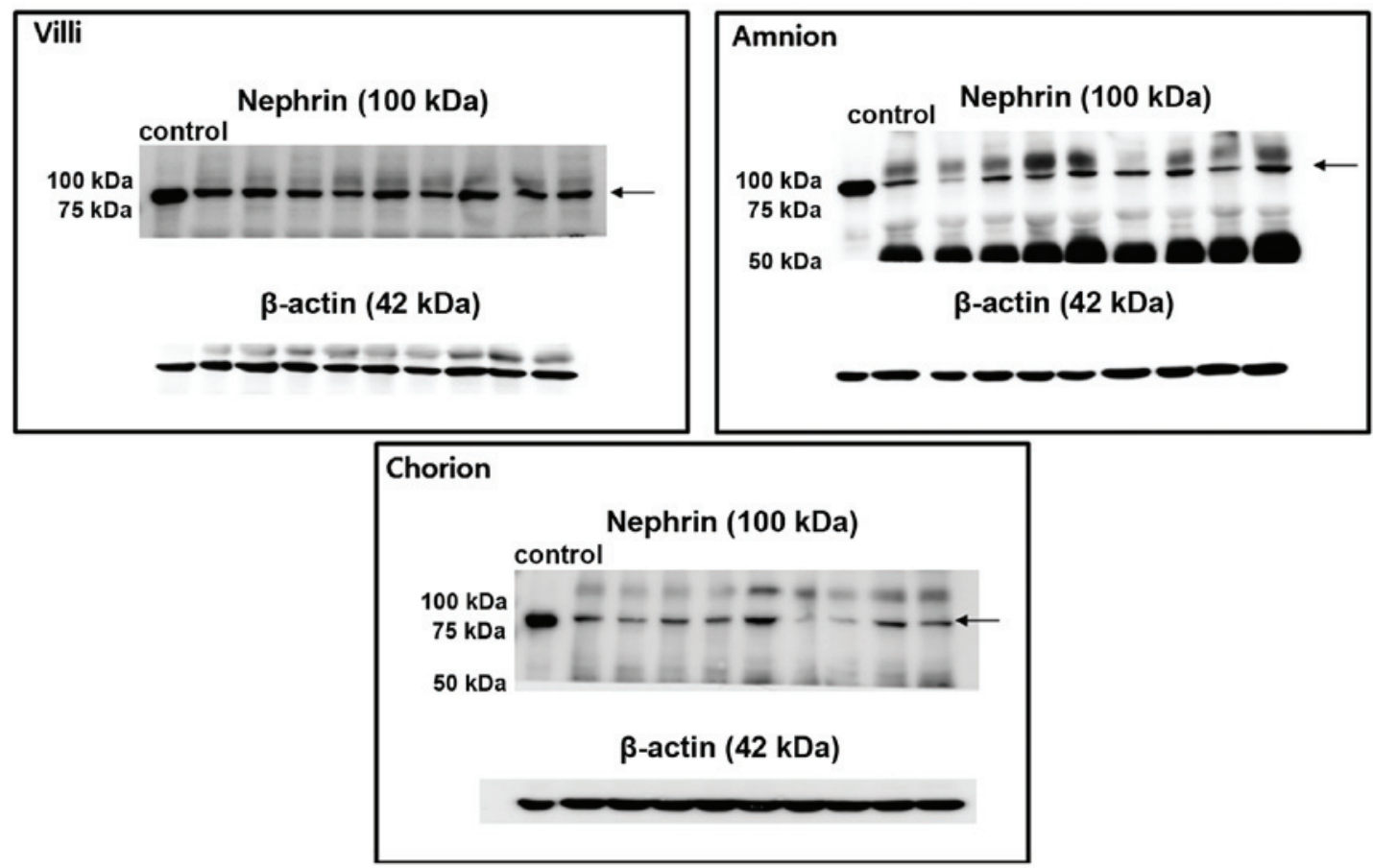

Figure 1. Expression of the nephrin gene in placental villi and fetal membranes. (A) Relative mRNA expression using reverse transcription-quantitative polymerase chain reaction $(\mathrm{n}=9)$. Data are expressed as the mean \pm standard deviation and are expressed as relative ratios of the expression levels of mRNA of the nephrin gene to those of GAPDH. (B) Western blot analysis for nephrin expression in the villi and fetal membranes (n=9).

(v/v)]. Following blocking with $5 \%$ goat serum for $1 \mathrm{~h}$, the cells were incubated with anti-nephrin antibody (polyclonal rabbit anti-human, 1:1,000; \#2265; ProSci) overnight at $4^{\circ} \mathrm{C}$. Negative controls consisted of cells incubated with rabbit $\operatorname{IgG}(1: 200$; cat no. ab27478; Abcam) at the same concentration as the antibody against nephrin. The cells were washed three times with PBS-T and incubated with goat polyclonal secondary antibody to rabbit $\operatorname{IgG}$ (1:200, DyLight ${ }^{\circledR}$ 594; cat no. ab96901; Abcam) for $1 \mathrm{~h}$. The cells were washed three times in PBS-T and mounted with a fluorescent mounting medium (Vector Laboratories, Inc., Burlingame, CA, USA). Fluorescent microscopy was performed using the LSM700 microscope (Carl Zeiss; Oberkochen, Germany).

Statistical analysis. Statistical analysis was performed using the SPSS software package, version 18.0 (IBM Inc., Armonk, NY, USA). The mRNA expression level of the nephrin gene was expressed as the mean \pm standard deviation. Descriptive statistics were used for the baseline characteristics and the linear mixed model for comparing the mRNA expression of the nephrin gene in the villi, chorion and amnion. $\mathrm{P}<0.05$ was considered to indicate a statistically significant difference.

\section{Results}

In all, nine healthy pregnant females were enrolled in the present study. The basal characteristics of the patients are shown in Table II. Due to the small sample size, data was expressed as the median and the range. One participant had a history of a previous cesarean section and concurrent myomectomy.

$m R N A$ and protein expression in the placenta. The expression of nephrin mRNA and protein in the uteroplacental unit were confirmed using RT-qPCR and western blotting, 

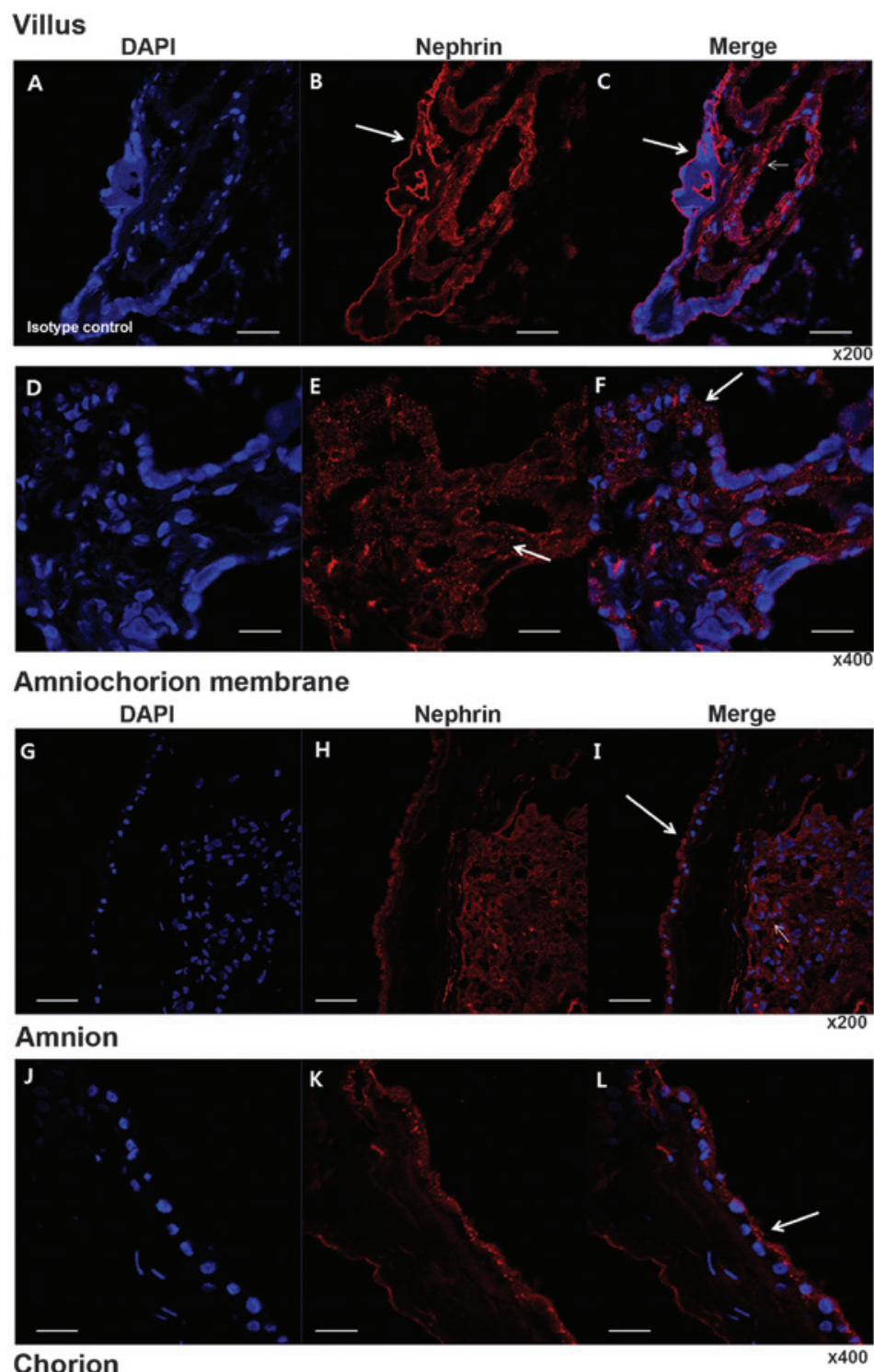

Chorion

O

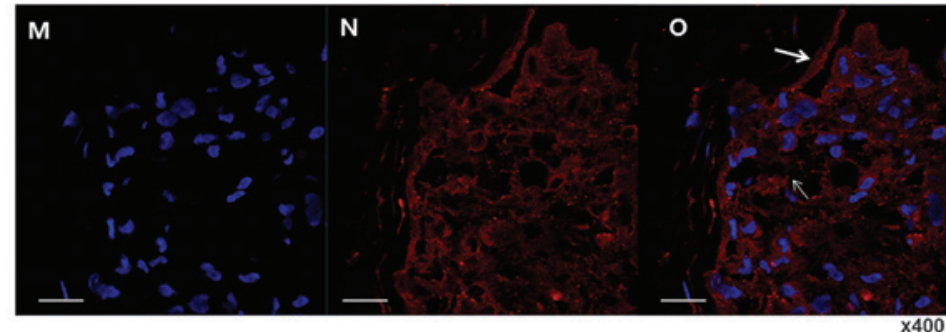

$\mathbf{N}$

$x 400$

Figure 2. Immunolocalization of nephrin in the placenta using immunofluorescence. (A-C) Immunofluorescence staining of isotype control with DAPI and nephrin in the villi. Magnification, x200. (D-F) Magnification, x400. Note that immunoreactive staining is distributed over almost all villous cytotrophoblasts (arrows in B, E and F) and endothelium of intravillous vessels (small arrow in E). (G-I) Immunofluorescence staining of isotype control with DAPI and nephrin in fetal membranes. Magnification, x200. (J-L) Immunofluorescence staining of isotype control with DAPI and nephrin in amnion. Magnification, x400. (M-O) Immunofluorescence staining of isotype control with DAPI and nephrin in chorion. Magnification, $\mathrm{x} 400$. A specific distribution at membranous lining (arrows in I, L and O), in addition to in fibroblasts in the chorion (small arrow in I and O). Note that specifically in the amnion, the outer lining of amniocytes was markedly positive (arrows in I and L). Bar $=50 \mu \mathrm{m}$. DAPI, 4'6-diamidino-2-phenylindole.

respectively (Fig. 1). The gene expression was relatively higher in the chorion when compared with the villus and amnion (mean, $2^{-\Delta \Delta \mathrm{Ct}} 3.79 \pm 4.49$ vs. $0.50 \pm 0.45$ and $2.12 \pm 2.41$ ); however, the difference was not statistically significant $(\mathrm{P}=0.37)$.

Immunolocalization of nephrin. Marked positive staining for nephrin was observed in the villi, chorion and amnion in the IF studies. Nephrin expression was clearly localized to the syncytiotrophoblast and endothelial cells of the arteries and veins of the chorionic plate, where the labeling was pronounced at the apical membrane of the syncytiotrophoblast. In the fetal membranes, intense immunoreactivity was localized to the cuboidal amniotic epithelium, particularly at the apical membrane and in the stromal cells (Fig. 2). 


\section{Discussion}

The main role of the placenta is the separation of maternal and fetal circulation, while also facilitating the transport of nutrients and substances from the mother to the fetus. As the placenta has extensive contact between the mother and the fetus in structures, such as the villus, amnion-chorion and the chorion-decidua, it is hypothesized that a filtering, barrier-like structure exists within the placenta at such interfaces. As hypothesized, evidence of the presence of nephrin in cells at the maternal-fetal interface was identified and immunoreactivity to nephrin was most prominent at the apical membrane of the syncytiotrophoblasts and amniotic epithelium, and in the stromal cells of the chorion. The present findings suggest the existence of a barrier-like structure formed by nephrin in the placenta similar to that of the glomerulus.

Although the present study did not investigate the function of nephrin in the placenta, it is likely that in the placenta, the protein may have a role similar to that observed in other organs (8-13). Its presence in astrocytes, which interact with the capillaries in the blood-brain barrier of the central nervous system suggested it exhibits a similar role as in the glomerular slit membrane (13). The role for nephrin as a barrier system has also been suggested in the testis due to the observation of nephrin in the Sertoli cells colocalized with zona occludens- 1 along the basement membrane of the seminiferous tubule in the testis (9). However, a previous study demonstrated that nephrin is expressed in the radial glial cells, which are involved in the directional migration of neurons and development of glial cell lineages (14), and that it binds with glutamate receptors and scaffolding molecules of the primary neuronal cells (5). Furthermore, its active role in the vesicle and actin interaction involved in insulin release was described in the pancreatic $\beta$ islet cells $(11,12)$. These findings suggest that nephrin not only functions as a barrier, but also exhibits functional roles, such as in cell maturation and development, cell-to-cell interaction and signaling (15-19).

It has been hypothesized that nephrin, expressed in the villi and the membranes holds such functions as well as other organs. Nephrin may be involved in the seclusion of fetal materials from the maternal circulation and thereby provide an immunologically anergic environment, which may be crucial to the exchange of nutrients between the mother and the fetus or it may have a role in placental development. If so, impairment of placental nephrin may result in an adverse pregnancy outcome, such as preeclampsia, restricted fetal growth or abnormal placental growth. As serum from a patient with preeclampsia has been revealed to damage the podocyte and shed nephrin via endothelin-mediated endothelin-1 (20), theoretically, nephrin in the syncytiotrophoblasts or the chorioamniotic membranes in close contact with the maternal serum may also be affected or disrupted via a similar mechanism. This may partly contribute to the pathophysiological mechanism of adverse fetal outcomes.

To the best of our knowledge, the present study is the first to provide evidence of the presence of nephrin in the human placenta and placental membranes. The identification of podocyte-associated nephrin expression in the placenta, particularly at the maternal-fetal interface, provides novel insight into the molecular basis of the selective permeability of the placental barrier, which requires further elucidation. The present findings highlight the requirement for further investigation into the placental expression of podocyte-associated proteins in pregnan- cies with complications and their specific roles in the human placenta.

\section{Acknowledgements}

This study was supported by the National Research Foundation of Korea funded by the Korean Government (grant no. 2011-0004069/7-2011-0208).

\section{References}

1. Tryggvason K and Wartiovaara J: Molecular basis of glomerular permselectivity. Curr Opin Nephrol Hypertens 10: 543-549, 2001.

2. Cooper ME, Mundel P and Boner G: Role of nephrin in renal disease including diabetic nephropathy. Semin Nephrol 22: 393-398, 2002.

3. Hennessy A and Makris A: Preeclamptic nephropathy. Nephrology (Carlton) 16: 134-143, 2011.

4. Son GH, Kim JH, Hwang JH, Kim YH, Park YW and Kwon JY: Urinary excretion of nephrin in patients with severe preeclampsia. Urinary nephrin in preeclampsia. Hypertens Pregnancy 30: 408-413, 2011.

5. Putaala H, Soininen R, Kilpeläinen P, Wartiovaara J and Tryggvason K: The murine nephrin gene is specifically expressed in kidney, brain and pancreas: inactivation of the gene leads to massive proteinuria and neonatal death. Hum Mol Genet 10: 1-8, 2001.

6. Beall MH, Amidi F, Gayle DA, Wang S, Beloosesky R and Ross MG: Placental and fetal membrane Nephrin and Neph1 gene expression: response to inflammation. J Soc Gynecol Investig 12: 298-302, 2005.

7. Welsh GI and Saleem MA: Nephrin-signature molecule of the glomerular podocyte? J Pathol 220: 328-337, 2010.

8. Aström E, Rinta-Valkama J, Gylling M, Ahola H, Miettinen A, Timonen T and Holthöfer H: Nephrin in human lymphoid tissues. Cell Mol Life Sci 63: 498-504, 2006.

9. Liu L, Aya K, Tanaka H, Shimizu J, Ito S and Seino Y: Nephrin is an important component of the barrier system in the testis. Acta Med Okayama 55: 161-165, 2001.

10. Putaala H, Sainio K, Sariola H and Tryggvason K: Primary structure of mouse and rat nephrin cDNA and structure and expression of the mouse gene. J Am Soc Nephrol 11: 991-1001, 2000.

11. Fornoni A, Jeon J and Varona Santos J, et al: Nephrin is expressed on the surface of insulin vesicles and facilitates glucose-stimulated insulin release. Diabetes 59: 190-199, 2010.

12. Rastaldi MP, Armelloni S, Berra S, Calvaresi N, Corbelli A, Giardino LA, Li M, Wang GQ, Fornasieri A, Villa A, et al: Glomerular podocytes contain neuron-like functional synaptic vesicles. FASEB J 20: 976-978, 2006.

13. Buniatian GH, Hartmann HJ, Traub P, Wiesinger H, Albinus M, Nagel W, Shoeman R, Mecke D and Weser U: Glial fibrillary acidic protein-positive cells of the kidney are capable of raising a protective biochemical barrier similar to astrocytes: expression of metallothionein in podocytes. Anat Rec 267: 296-306, 2002.

14. Li M, Armelloni S, Ikehata M, Corbelli A, Pesaresi M, Calvaresi N, Giardino L, Mattinzoli D, Nistico F, Andreoni S, et al: Nephrin expression in adult rodent central nervous system and its interaction with glutamate receptors. J Pathol 225: 118-128, 2011.

15. Grant SG, O'Dell TJ, Karl KA, Stein PL, Soriano P and Kandel ER: Impaired long-term potentiation, spatial learning, and hippocampal development in fyn mutant mice. Science 258: 1903-1910, 1992.

16. Huber TB and Benzing T: The slit diaphragm: a signaling platform to regulate podocyte function. Curr Opin Nephrol Hypertens 14: 211-216, 2005.

17. Li M, Armelloni S, Edefonti A, Messa P and Rastaldi MP: Fifteen years of research on nephrin: what we still need to know. Nephrol Dial Transplant 28: 767-770, 2013.

18. Verma R, Wharram B, Kovari I, Kunkel R, Nihalani D, Wary KK, Wiggins RC, Killen P and Holzman LB: Fyn binds to and phosphorylates the kidney slit diaphragm component Nephrin. J Biol Chem 278: 20716-20723, 2003.

19. Wagner N, Morrison H, Pagnotta S, Michiels JF, Schwab Y, Tryggvason K, Schedl A and Wagner KD: The podocyte protein nephrin is required for cardiac vessel formation. Hum Mol Genet 20: 2182-2194, 2011.

20. Collino F, Bussolati B, Gerbaudo E, et al: Preeclamptic sera induce nephrin shedding from podocytes through endothelin-1 release by endothelial glomerular cells. Am J Physiol Renal Physiol 294: F1185-F1194, 2008. 